\title{
Preoperative echocardiographic predictors for 1-year mortality in patients treated with standard endovascular aneurysm repair for abdominal aortic aneurysm
}

\author{
Tomas Ohrlander ${ }^{1}$, Magnus Dencker ${ }^{2}$, Stefan Acosta ${ }^{3}$ \\ ${ }^{1}$ Department of Surgery, Eksjö County Hospital, Eksjö, Sweden \\ ${ }^{2}$ Department of Clinical Physiology and Nuclear Medicine, Vascular Center, Malmö, Sweden \\ ${ }^{3}$ Lund and Skåne University Hospital, Malmö, Sweden \\ Email: stefan.acosta@telia.com
}

Received 4 April 2013; revised 10 May 2013; accepted 19 May 2013

Copyright (C) 2013 Tomas Ohrlander et al. This is an open access article distributed under the Creative Commons Attribution License, which permits unrestricted use, distribution, and reproduction in any medium, provided the original work is properly cited.

\begin{abstract}
Background: Abdominal aortic aneurysm (AAA) and cardiovascular disease are intimately associated, the latter representing the most common cause of death in Sweden. Cardiac complications are held responsible for the majority of perioperative morbidity and mortality in patients undergoing repair of AAA. The importance of preoperative thorough cardiac assessment is therefore obvious. The aim of this study was to evaluate the prognostic significance of preoperative echocardiographic findings for 1-year mortality after elective endovascular aneurysm repair (EVAR) of infrarenal AAA. Design: Retrospective analysis. Methods: The 505 patients were identified in a prospective database for endovascular interventions between 1998 and 2011, and data were retrieved from patient records. Preoperative echocardiography reports in $\mathbf{3 8 0}$ patients were reviewed and findings were notified according to a predefined protocol. Results: The 1-year mortality rate was $6.7 \%$. Severe valve disease was present in $8.7 \%$ of the patients, aortic valve stenosis being the leading cause of valve pathology. Severe valve disease (OR 3.5, 95\% CI [1.2 $10.7] ; p=0.025)$ and chronic kidney disease grade $\geq 3$ (OR 7.5, 95\% CI [2.1 - 26.1]; $p=0.002)$ were the only independent risk factors for increased mortality rate at 1-year. Conclusion: Echocardiography should be a part of the preoperative workup in AAA patients. Finding of severe valve disease should be further evaluated by a cardiologist prior to EVAR.
\end{abstract}

Keywords: Echocardiography; EVAR; Abdominal Aortic Aneurysm; Valve Disease; Aortic Valve Stenosis

\section{INTRODUCTION}

Abdominal aortic aneurysm (AAA) may be considered as one of many end-stage manifestations of atherosclerotic cardiovascular disease and it is the most common cause of death in Sweden [1]. Screening for asymptomatic AAA has been questioned since difficulties in reducing all-cause mortality in this patient group is evident $[2,3]$. Cardiac complications represent the major cause of peri-operative morbidity and mortality in patients undergoing vascular surgery [4] and the importance of thorough cardiac preoperative assessment as a part of preoperative patient selection seems to be an important factor for reducing mortality in this patient group.

Echocardiography has been a standard tool for preoperative cardiac assessment prior to endovascular repair (EVAR) for AAA in our and other institutions. In a previous report from our institution, we were able to show that only ejection fraction staging were associated with long term mortality in a univariate but not in a multivariate analysis [5]. Thus, the need for an extended study to further evaluate the possible influence of preoperative echocardiographic findings in EVAR patients seemed warranted. The aim of this study was to evaluate the prognostic value of preoperative echocardiographic findings for 1-year mortality after standard infrarenal EVAR.

\section{METHODS}

\subsection{Patients}

The Vascular Centre, Malmö-Lund is a tertiary referral centre for patients suffering from vascular diseases. This study comprised patients who underwent elective, nonfenestrated EVAR (Zenith ${ }^{\circledR}$ stentgrafts [Cook Europe A/S, Bjaeverskov, Denmark]) for infrarenal non-ruptured 
AAA between May 1998 and May 2011. Four hundred and seventy-five bifurcated, 27 aorto-uniiliac and femoral-femoral crossover bypasses and two aorto-aortic stentgrafts were inserted. One per-operative conversion to open surgery was performed. Preoperative work-up protocol included mini-spirometry and echocardiography between 1998-2006. In the latter time period between 2007-2011 these investigations were performed according to angiologists decision. The proportion of preoperative echocardiography performed in the former and the latter time period was $83.9 \%(255 / 304)$ and $62.2 \%$ $(125 / 201)$, respectively. The patients were identified in the prospective database for endovascular interventions and complementary data were retrieved from patient records. This study was ethically approved by the Research Ethics Committee, University of Lund.

\subsection{Electrocardiography}

As part of the preoperative evaluation, electrocardiography (ECG) at rest was performed to assess signs of myocardial ischemia and atrial fibrillation. Evidence of myocardial ischemia (ECG ischemia) were defined as presence of pathological Q/QS patterns suggestive of previous myocardial infarction, deep or moderate $\mathrm{T}$ wave inversion, ST-segment depression or complete left bundle branch block [6,7].

\subsection{Echocardiography}

Preoperative transthoracic echocardiographic examinations were carried out with Sonos 2500 or Sonons 5500 (Philips, Eindhoven, the Netherlands), by highly trained personal. Left ventricular ejection fraction $(\mathrm{EF})$, regional wall abnormalities and presence of left ventricular hypertrophy (LVH) were assessed visually $[8,9]$. Subsequently EF was graded as normal $(\geq 55 \%)$, mildly reduced $(45 \%-50 \%)$, moderately reduced $(35 \%-40 \%)$ or severely reduced $(<35 \%)[8,9]$. Aortic valve stenosis was defined as a maximal systolic velocity $>2.5 \mathrm{~m} / \mathrm{s}$; the velocity ratio was used if EF was reduced [10]. Aortic and mitral valve insufficiencies were graded as mild, moderate or severe [11]. Severe valve disease was defined as presence of aortic valve stenosis, aortic valve insufficiency ( $\geq$ moderate) or mitral valve insufficiency ( $\geq$ moderate). No patient had mitral valve stenosis.

\subsection{Evaluation of Renal Function}

In accordance with the Cockroft-Gault formula, glomerular filtration rate (GFR) was calculated-comprising serum creatinine, age, weight and gender [12]. Stages of chronic kidney disease were determined by GFR levels set by the National Kidney Foundation guidelines [13]: Stage I was defined as GFR $>90 \mathrm{ml} / \mathrm{min} / 1.73 \mathrm{~m}^{2}$ (nor- mal or increased GFR), stage II as GFR $60-89 \mathrm{ml} / \mathrm{min} /$ $1.73 \mathrm{~m}^{2}$ (minute decrease in GFR), stage III as GFR 30 $59 \mathrm{ml} / \mathrm{min} / 1.73 \mathrm{~m}^{2}$ (moderate decrease in GFR), stage IV as GFR 15 - $29 \mathrm{ml} / \mathrm{min} / 1.73 \mathrm{~m}^{2}$ (substantial decrease in GFR) and stage $\mathrm{V}$ as GFR $<15 \mathrm{ml} / \mathrm{min} / 1.73 \mathrm{~m}^{2}$ (endstage renal disease in need of dialysis).

\subsection{Spirometry}

Spirometry was performed in accordance with standards set by the European Respiratory Society [14]. The majority of the spirometries were performed at the Vascular Center, Malmö-Lund, and the spirometer (Mirolab 3000 or EasyOne) was utilized under the supervision of a physiotherapist (mini-spirometry), measuring forced expiratory volume in one second (FEV1) and forced vital capacity (FVC). $\mathrm{FEV}_{1} \%$ was defined as $\mathrm{FEV}_{1}$ in relation to predicted normal $\mathrm{FEV}_{1}$, and $\mathrm{FVC} \%$ as $\mathrm{FVC}$ in relation to predicted normal FVC [15-17]. The presence and severity of chronic obstructive pulmonary disease (COPD) was graded in accordance with the Global Initiative for Chronic Obstructive Lung Diseases (GOLD) guidelines [18]. COPD was present if $\mathrm{FEV}_{1} / \mathrm{FVC}<70 \%$ (age < 65) or $\mathrm{FEV}_{1} / \mathrm{FVC}<65 \%$ (age $\geq 65$ ). Stage 1 (mild) was defined as $\mathrm{FEV}_{1} \geq 80 \%$ of predicted value, Stage 2 (moderate) as $\mathrm{FEV}_{1} \geq 50 \%-<80 \%$, Stage 3 (severe) as $\mathrm{FEV}_{1} \geq 30 \%-<50 \%$ and Stage 4 (very severe) as $\mathrm{FEV}_{1}$ $<30 \%$ of predicted value.

\subsection{Follow up}

All patients were monitored from the day of EVAR until day of death or May 25, 2012. Median time of follow-up was 48 (IQR 25 - 85) Mortality data were retrieved from the Swedish Population Registry.

\subsection{Other Definitions}

The shortest transverse diameter of the AAA at its widest portion was defined as the maximal AAA diameter. Hypertension was considered if the patient was on antihypertensive medication or had previously been diagnosed with hypertension. Cerebrovascular disease was taken into consideration if the patient had suffered a stroke (cerebral bleeding or infarction) or transient ischemic attack (TIA). In patients with a history of myocardial infarction, angina pectoris, coronary artery bypass grafting or percutaneous coronary angioplasty, ischemic heart disease was taken into count. Diabetes mellitus was noted if the patients were on any form of anti-diabetic treatment (diet, oral hypoglycaemic agents, or insulin). Anaemia was defined as haemoglobin $<134 \mathrm{~g} / \mathrm{L}$ in men, and $<117 \mathrm{~g} / \mathrm{L}$ in women. Both current and former smokers were classified as smokers. 


\subsection{Statistical Methods}

SPSS 17.0 software (SPSS Inc, Chicago, Ill) was used for data managing and statistical analysis. Differences in proportions were evaluated using the chi-square test. Mann-Whitney U tests were used when comparing groups using continuous variables. Correlations were calculated with the Pearson or Spearman's rank test. Variables associated with mortality at 1 year were further tested in a binary multi-variate regression model and associations were expressed in Odds ratios (OR) with 95\% confidence intervals (CI). Difference in long-term mortality between patients undergoing echocardiography versus those who did not was evaluated with a univariate Cox regression analysis and was expressed in terms of hazard ratio (HR) with 95\% confidence interval (CI) and pvalue. $\mathrm{p}<0.05$ was considered significant.

\section{RESULTS}

\subsection{Patient Characteristics}

Median age among the 505 patients was 74 years (IQR 70 - 79). The proportion of women was 14\% (73/505). Median age among women and men was 75 years (IQR 71 - 78) and 74 years (IQR 69 - 79), respectively ( $\mathrm{p}=$ 0.61 ). The body mass index among men and women were 25.4 (IQR 23.1 - 28.2) and 26.1 (IQR 22.8 - 29.0), respectively $(\mathrm{p}=0.41)$. The proportion of smokers was $87 \%(401 / 460)$. The frequency of hypertension, diabetes mellitus, ischemic heart disease, cerebro-vascular disease and peripheral arterial occlusive disease were $84 \%, 12 \%$, $44 \%, 16 \%$ and $14 \%$, respectively. The median size of the maximal abdominal aortic aneurysm diameter among men and women were $60 \mathrm{~mm}$ (IQR 55 - 70) and $57 \mathrm{~mm}$ $(53-61)$, respectively $(p<0.001)$.

\subsection{Preoperative Cardiovascular Medications}

The proportion of patients on any cardiovascular medication was $86 \%(435 / 505)$. The frequency of specific medications were as follows: Acetyl salicylic acid (52\%), statins (48\%), warfarin (9\%), Calcium antagonist $(25 \%)$, diuretics $(32 \%)$, digitalis $(6 \%)$, beta blockers $(45 \%)$, angiotensinogen converting enzyme inhibitors (27\%) and angiotensinogen receptor II blockers (11\%).

\subsection{Preoperative Echocardiographic Findings}

The echocardiographic findings are shown in Table $\mathbf{1 .}$ Severe valve disease was present in 33 patients and the specific valve pathologies among these were as follows: Mitral valve insufficiency $(n=9)$, aortic valve stenosis, aortic valve insufficiency and mitral valve insufficiency $(n=7)$, aortic valve stenosis $(n=6)$, aortic valve stenosis and mitral valve insufficiency $(\mathrm{n}=3)$, aortic valve insuf-
Table 1. Echocardiographic findings prior to EVAR in 380 patients.

\begin{tabular}{lc}
\hline Variable & N (\%) of all eligible patients \\
\hline Ejection fraction staging & $299(78.7)$ \\
Normal & $47(12.4)$ \\
Slightly decreased & $17(4.5)$ \\
Moderately decreased & $17(4.5)$ \\
Very decreased & $90(23.7)$ \\
Regional or global hypokinesia & $83(21.8)$ \\
Left ventricular hypertrophy & \\
Valve disease & $26(6.8)$ \\
Aortic valve stenosis & $76(20.0)$ \\
Aortic valve insufficiency & $76(20.0)$ \\
Mitral valve insufficiency & $140(36.8)$ \\
Any valve disease & $33(8.7)$ \\
Any severe valve disease &
\end{tabular}

ficiency and mitral valve insufficiency $(\mathrm{n}=3)$, aortic valve insufficiency $(\mathrm{n}=3)$, and aortic valve stenosis and aortic valve insufficiency $(n=2)$. The leading cause(s) of valve pathology in patients with severe valve disease were aortic valve stenosis $(\mathrm{n}=18)$, mitral valve insufficiency $(\mathrm{n}=9)$, aortic valve insufficiency $(\mathrm{n}=3)$, and aortic valve and mitral valve insufficiencies $(n=3)$. EF among patients with severe valve disease (median EF of $55 \%$ [IQR 40 - 55]) was lower ( $\mathrm{p}<0.001)$, compared to those with non-severe valve disease (median EF of $55 \%$ [IQR 55 - 55]). Hypokinesia was correlated with lower EF $(r=-0.71 ; p<0.001)$, presence of ECG ischemia $(r=$ $0.32 ; \mathrm{p}<0.001)$, medication with digitalis $(\mathrm{r}=0.26 ; \mathrm{p}=$ $0.008)$ and medication with diuretics $(r=0.12 ; \mathrm{p}=$ $0.016)$.

\subsection{Comparison of Co-Morbidity Variables among Patients Who Underwent Preoperative Echocardiography and Those Who Did Not}

The percentage of statin therapy was found to be higher among patients that did not undergo preoperative echocardiography versus those who did (Table 2). There was no difference in 1-year mortality among patients undergoing echocardiography versus those who did not.

\subsection{Risk Factors Associated with 1-Year Mortality after EVAR}

The 30-day and 1-year mortality rate were $2.4 \%$ and 6.7 $\%$, respectively. The co-morbidity severity predictors for 
Table 2. Comparison of co-morbidiy variables among patients who underwent preoperative echocardiography versus those who did not.

\begin{tabular}{lccc}
\hline & Echocardiography (\%) & No echocardiography (\%) & p \\
\hline Patients & 380 & 125 & $31 / 125(24.8)$ \\
Age $\geq 80$ years & $74 / 380(19.5)$ & $15 / 125(12.0)$ & 0.20 \\
Women & $58 / 380(15.3)$ & & 0.37 \\
Preoperative medication & & $72 / 125(57.6)$ & 0.17 \\
Acetyl salicylic acid & $192 / 380(50.5)$ & $73 / 125(58.4)$ & 0.009 \\
Statins & $170 / 379(44.9)$ & $34 / 125(27.2)$ & 0.23 \\
Diuretics & $125 / 380(32.9)$ & $45 / 123(36.6)$ & 0.67 \\
Anemia & $131 / 380(34.5)$ & $47 / 121(38.8)$ & 0.096 \\
Chronic kidney disease, grade $\geq 3$ & $180 / 379(47.5)$ & $38 / 120(31.7)$ & 0.30 \\
Myocardial ischemia on ECG & $134 / 363(36.9)$ & $7 / 43(16.3)$ & 0.34 \\
COPD, grade $\geq 3$ & $35 / 311(11.3)$ & $8 / 125(6.4)$ & 0.86 \\
1-year mortality & $26 / 379(6.9)$ & & \\
\hline
\end{tabular}

1-year mortality are shown in Table 3. Chronic kidney disease, grade $\geq 3$ (OR 7.5, 95\% CI [2.1 - 26.1]; $\mathrm{p}=$ 0.002 ) and severe valve disease (OR 3.5, 95\% CI [1.2 10.7 ]; $p=0.025$ ) were independent predictors for mortality at 1 -year.

\subsection{Long-Term Mortality after EVAR}

Mortality during a median follow up time of 48 months was $44.6 \%$. There was no difference in long-term mortality between those who underwent echocardiography versus those who did not (HR 0.82, 95\% CI [0.58 - 1.14]; $\mathrm{p}=0.23$ ).

\section{DISCUSSION}

The most important finding of the present study was that patients with severe valve disease undergoing elective standard infrarenal EVAR for AAA had, after multivariable testing, an increased risk of mortality at 1 -year follow-up. In a previous report [3], the presence of a valvular heart disease and a positive dobutamine stress echocardiography were found to be the only factors that were independently associated with increased 30-day cardiovascular morbidity after EVAR and open repair of AAA. In particular, aortic valve stenosis in the present study was found to be the most common cause of severe valve disease and its presence had the highest 1-year mortality rate in comparison with the other valvular diseases. A pressure gradient $>40 \mathrm{mmHg}$ across the aortic valve has been found to be independently associated with increased risk of myocardial infarction and cardiogenic pulmonary edema after major elective non-cardiac sur- gery [19] and case-control studies have shown a clear association between aortic valve stenosis and non-fatal myocardial infarction $[20,21]$ after elective non-cardiac surgery. In their guideline, American College of Cardiologists and American Heart Association, firmly states that severe aortic valve disease poses the greatest risk for non-cardiac surgery [22] and that elective non-cardiac surgery should be postponed or canceled in symptomatic patients with aortic valve disease or in asymptomatic patients with severe valve disease if the valve has not been evaluated within the year. Patients with symptommatic aortic valve disease require aortic valve replacement before any necessary elective non-cardiac surgery [23] to avoid a surgical mortality risk of $10 \%$. Even though it seems that the cardiovascular response after EVAR, in comparison to open repair of AAA, may be less pronounced [24-26], the present study mortality data support the view that patients with severe valve disease, particularly aortic valve stenosis, should be referred to a cardiologist for medical optimization and in case of aortic valve stenosis, considering referral to the cardio-thoracic surgeon for aortic valve replacement.

In these days of screening programs of AAA and detection of a large proportion of small AAAs, it is most likely that a higher proportion of patients will undergo elective repair and a lower proportion acute repair. Hence, timely preoperative risk stratification will take a larger place in the management of patients with AAA. Unfortunately, preoperative screening, by any physician, for audible murmurs emanating from the heart and its association with severe valve disease at clinical examination seems to be an inaccurate tool [27], whereas 
Table 3. Co-morbidity severity predictors for 1-year mortality after standard EVAR for AAA.

\begin{tabular}{|c|c|c|c|c|c|}
\hline & & & & \multicolumn{2}{|c|}{ Multivariate analysis } \\
\hline & Eligible patients (\%) & 1-year Mortality (\%) & p-value & OR $(95 \% \mathrm{CI})$ & p-value \\
\hline All patients & 505 & $34 / 504(6.7)$ & & & \\
\hline Age $\geq 80$ years & $105 / 504(20.8)$ & 9/105 (8.6) & 0.40 & & \\
\hline \multicolumn{6}{|l|}{ Preoperative medication } \\
\hline Acetyl salicylic acid & $264 / 505(52.3)$ & $19 / 263(7.2)$ & 0.66 & & \\
\hline Statins & $243 / 504(48.2)$ & $13 / 242(5.4)$ & 0.23 & & \\
\hline Anemia $^{*}$ & $176 / 502(35.1)$ & $18 / 176(10.2)$ & 0.015 & $0.9(0.4-2.3)$ & 0.87 \\
\hline Chronic kidney disease grade $\geq 3^{*}$ & $227 / 499(45.5)$ & $26 / 227(11.5)$ & $<0.001$ & $7.5(2.1-26.1)$ & 0.002 \\
\hline Myocardial ischemia on $\mathrm{ECG}^{*}$ & $172 / 482(35.7)$ & $17 / 172(9.9)$ & 0.021 & $1.8(0.7-4.7)$ & 0.21 \\
\hline Ejection fraction $<55 \%{ }^{*}$ & $81 / 379(21.4)$ & $10 / 81(12.3)$ & 0.039 & $1.0(0.2-4.9)$ & 1.0 \\
\hline Regional or global hypokinesia $^{*}$ & 90/379 (23.7) & $11 / 90(12.2)$ & 0.031 & $1.2(0.3-5.6)$ & 0.77 \\
\hline Aortic valve stenosis & $26 / 379(6.9)$ & $5 / 26(19.2)$ & 0.013 & & \\
\hline Mitralis valve insufficiency & $76 / 379(20.1)$ & $11 / 76(14.5)$ & 0.005 & & \\
\hline Any valve disease & $140 / 379(36.9)$ & $15 / 140(10.7)$ & 0.038 & & \\
\hline Severe valve disease ${ }^{*}$ & $33 / 379(8.7)$ & $7 / 33(21.2)$ & 0.001 & $3.5(1.2-10.7)$ & 0.025 \\
\hline COPD, stage $\geq 3$ & 42/354 (11.9) & $2 / 42(4.8)$ & 0.79 & & \\
\hline
\end{tabular}

*variables entered in multi-variate analysis.

echocardiography identifies severe valve disease, gives valuable diagnostic information for better medical therapy and is a prognostic tool.

The severe valve disease was not an unusual echocardiographic finding among AAA patients in the present study, approximately in a frequency of nine percent, which is similar to the $10 \%$ rate reported in another study using similar criteria for severe valve disease. The other way around, performing echocardiography first followed by immediate ultrasound of the aorta, has also been suggested in elderly patients with cardiovascular disease referred for echocardiographic examination [28,29], since the same transducer fits well for examination of the abdominal aorta and since the investigation in trained hands is simple, accurate and fast $[28,29]$. The percentage of patients with an AAA in these selected patient series may be up to $6.5 \%$ [30].

One limitation of the study was that preoperative echocardiographic evaluation was not performed in all study patients and that standardized echocardiography was not included in the workup during the latter part of the study. Therefore a comparative analysis was performed between those undergoing echocardiography versus those who did not (Table 2). There was no difference in short or long-term mortality between the two groups. It was only found that medication with statin therapy at admission was more common among those patients not scheduled for preoperative echocardiography, a logical finding since these patients were often entered during the latter part of the study when medical activity was higher.

In conclusion, preoperative echocardiographic finding of a severe valve disease in patients undergoing standard EVAR for AAA was found to be an independent predicttor for 1-year mortality. Echocardiography should be included in the preoperative workup in AAA patients, and finding of a severe valve disease should lead to consultation by a cardiologist prior to EVAR.

\section{REFERENCES}

[1] Dödsorsaker 2010. http://www.socialstyrelsen.se/Lists/Artikelkatalog/Attach ments/18394/2011-7-6.pdf

[2] Van der Graaf, Y. (2008) Aggressive vascular surgery in asymptomatic patients is a bad idea. British Journal of Surgery, 95, 671-673. doi:10.1002/bjs.6233

[3] Earnshaw, J.J. (2011) Doubts and dilemmas over abdominal aortic aneurysm. British Journal of Surgery, 5, 607-608. doi:10.1002/bjs.7495

[4] Troisi, N., Dorigo, W., Lo Sapio, P., Pratesi, G., Pulli, R., Gensini, G.F., et al. (2010) Preoperative cardiac assessment in patients undergoing aortic surgery: Analysis of 
factors affecting outcomes. Annals of Vascular Surgery, 24, 733-740. doi:10.1016/j.avsg.2010.01.009

[5] Ohrlander, T., Dencker, M., Dias, N.V., Gottsäter, A. and Acosta, S. (2011) Cardiovascular predictors for long-term mortality after EVAR for AAA. Vascular Medicine, 16, 422-427. doi:10.1177/1358863X11425713

[6] MacFarlane, P.W. (2000) Minnesota coding and the prevalence of ECG abnormalities. Heart, 84, 582-584. doi:10.1136/heart.84.6.582

[7] De Bacquer, D., de Backer, G. and Kornitzer, M. (2000) Prevalences of ECG findings in large population based samples of men and women. Heart, 84, 625-633. doi:10.1136/heart.84.6.625

[8] Willenheimer, R., Erhardt, L. and Dahlöf, B. (1999) Simplified echocardiography: An accurate and inexpensive method for the assessment of left ventricular hypertrophy. European Heart Journal, 20, 1437-1438. doi:10.1053/euhj.1999.1656

[9] Gudmundsson, P., Rydberg, E., Winter, R. and Willenheimer, R. (2005) Visually estimated left ventricular ejection fraction by echocardiography is closely correlated with formal quantitative methods. International Journal of Cardiology, 101, 209-212. doi:10.1016/j.ijcard.2004.03.027

[10] Baumgartner, H., Hung, J., Bermejo, J., Chambers, J.B., Evangelista, A., Griffin, B.P., et al. (2009) EAE/ASE. Echocardiographic assessment of valve stenosis: EAE/ ASE recommendations for clinical practice. European Journal of Echocardiography, 10, 1-25. doi:10.1093/ejechocard/jen303

[11] Gottdiener, J.S., Panza, J.A., St John Sutton, M., Bannon, P., Kushner, H. and Weissman, N.J. (2002) Testing the test: The reliability of echocardiography in the sequential assessment of valvular regurgitation. American Heart Journal, 144, 115-121. doi:10.1067/mhj.2002.123139

[12] Cockcroft, D.W. and Gault, M.H. (1976) Prediction of creatinine clearance from serum creatinine. Nephron, 16, 31-41. doi:10.1159/000180580

[13] Abboud, H. and Henrich, W. (2010) Stage IV chronic kidney disease. The New England Journal of Medicine, 362, 56-65. doi:10.1056/NEJMcp0906797

[14] Quanjer, H., Tammeling, G.J., Coles, J.E., Pedersen, O.F., Peslin, R. and Yernault, J.-C. (1993) Lung volumes and forced ventilatory flows. Report working party. Standardization of Lung function tests. European community for steel and coal. Official statement of the European respiratory society. European Respiratory Journal, 6, 5-40.

[15] Berglund, E., Birath, G., Bjure, J. Grimby, G., Kjellmer, I. and Sandqvist, L., et al. (1963) Spirometric studies in normal subjects. I. Forced expirograms in subjects between 7 and 70 years of age. Acta Medica Scandinavica, 173, 185-192.

[16] Birath, G., Kjellmer, I. and Sandqvist, L. (1963) Spirometric studies in normal subjects. II. Ventilatory capacity tests in adults. Acta Medica Scandinavica, 173, 193-198.

[17] Grimby, G. and Soderholm, B. (1963) Spirometric studies in normal subjects. III Static lung volumes and maximum voluntary ventilation in adults with note on physical fit- ness. Acta Medica Scandinavica, 173, 199. doi:10.1111/j.0954-6820.1963.tb16523.x

[18] Pauwels, R.A., Buist, A.S., Calverley, P.M., Jenkins, C.R. and Hurd, S.S. (2001) Global strategy for the diagnosis, management and prevention of chronic obstructive pulmonary disease. NHLBI/WHO Global Initiative for Chronic Obstructive Lung Disease (GOLD). Workshop summary. American Journal of Respiratory and Critical Care Medicine, 163, 1256-1276. http://www.goldcopd.com.

[19] Rohde, L., Polanczyk, C., Goldman, L., Cook, F., Lee, R. andLee, T. (2001) Usefulness of transthoracic echocardiography as a tool for risk stratification of patients undergoing major noncardiac surgery. American Journal of Cardiology, 87, 505-509. doi:10.1016/S0002-9149(00)01421-1

[20] Zahid, M., Sonel, A., Saba, S. and Good, C. (2005) Perioperative risk of non-cardiac surgery associated with aortic stenosis. American Journal of Cardiology, 96, 436438. doi:10.1016/j.amjcard.2005.03.095

[21] Kertai, M.D., Bountioukos, M., Boersma, E., Bax, J.J., Thomsaon, I.R., Sozz, F., et al. (2004) Aortic stenosis: An underestimated risk factor for perioperative complications in patients undergoing non-cardiac surgery. American Journal of Medicine, 116, 8-13. doi:10.1016/j.amjmed.2003.07.012

[22] Lee, F., Beckman, J., Brown, K., Calkins, H., Chaikof, E., Fleishmann, K., et al. (2007) ACC/AHA 2007 guideline on perioperative cardiovascular evaluation and care for noncardiac surgery: A report of the American College of Cardiology/American Heart Association Task Force on Practical Guidelines. Circulation, 116, e418-e500.

[23] Otto, C.M. (2006) Valvular stenosis: Disease severity and timing of intervention. Journal of the American College of Cardiology, 47, 2141-2151. doi:10.1016/i.jacc.2006.03.002

[24] Cuypers, P.W.K., Gardien, M., Buth, C., Peels, C.H., Charbon J.A. and Hop, W.C.J. (2001) Randomized study comparing cardiac response in endovascular and open abdominal aortic aneurysm repair. British Journal of Surgery, 88, 1059-1065. doi:10.1046/j.0007-1323.2001.01834.x

[25] Brown, L.C., Thompson, S.G., Greenhalgh, R.M. and Powell, J.T. (2011) Incidence of cardiovascular events and death after open or endovascular repair of abdominal aortic aneurysm in the randomized EVAR trial 1. British Journal of Surgery, 98, 935-942. doi:10.1002/bjs.7485

[26] Lederle, F., Freischlag, J., Kyriakides, T., Matsumura, J., Padberg, F., Kohler, T., et al. (2012) Long-term comparison of endovascular and open repair of abdominal aortic aneurysm. The New England Journal of Medicine, 367, 1988-1997. doi:10.1056/NEJMoa1207481

[27] Loxdale, S.J., Sneyd, J.R., Donovan, A., Werrett, G. and Viira, D.J. (2012) The role of routine pre-operative bedside echocardiography in detecting aortic stenosis in patients with a hip fracture. Anaesthesia, 67, 51-54. doi:10.1111/j.1365-2044.2011.06942.x

[28] Navas, E., McCalla-Lewis, A., Fernandez Jr., B., Pinski, S., Novaro, G. and Asher, C. (2012) Abdominal aortic aneurysm screening during transthoracic echocardiogra- 
phy: Cardiologist and vascular medicine specialist interpretation. World Journal of Cardiology, 26, 31-35. doi:10.4330/wjc.v4.i2.31

[29] Aboyans, V., Kownator, S., Lafitte, M., Brochet, E., Emmerich, J., Tribouilloy, C., et al. (2010) Screening abdominal aortic aneurysm during echocardiography: Literature review and proposal for a French nationwide study. Archives of Cardiovascular Disease, 103, 552-558.
[30] Spittell, P.C., Ehrsam, J.E., Andersson, L. and Seward, J.B. (1997) Screening for abdominal aortic aneurysm during transthoracic echocardiography in a hypertensive patient population. Journal of the American Society of Echocardiography, 10, 722-727.

doi:10.1016/S0894-7317(97)70115-9 